\title{
Optical-Terahertz Biphotons
}

\author{
G.Kh. Kitaeva \\ Lomonosov Moscow State University, Moscow, Russia, gkitaeva@physics.msu.ru
}

The quantum correlated pairs of photons, usually named as biphotons, are the well known quantum optical objects, comprehensively studied and exploited in modern quantum information schemes [1]. Biphotons are easily generated as pure quantum states in the process of parametric down-conversion (PDC) of optical laser radiation in a quadratic nonlinear medium, and serve further as a basis for preparation of different types of entangled photons and squeezed vacuum states. During PDC each pump photon of frequency $\omega_{p}$ decays into a pair of photons: a signal photon of frequency $\omega_{s} \geq \omega_{p} / 2$ and an idler photon of lower frequency $\omega_{i} \leq \omega_{p} / 2$. Formally, only one restriction is imposed on specific values of $\omega_{i}$ and $\omega_{s}$. It follows from the energy conversation law $\hbar \omega_{i}+\hbar \omega_{s}=\hbar \omega_{p}$, so that the biphotons can be generated in a wide spectral range. Up to now PDC processes are studied mostly in the frequency-degenerate cases, when $\omega_{s}=\omega_{i}=\omega_{p} / 2$, or slightly nondegenerate ones, when the difference between signal and idler frequencies is less than the spectral band of optical transmission of the nonlinear medium. We propose to consider properties of biphotons with extremely different frequencies, when the signal photon frequency is just close to $\omega_{p}$, while the idler photon frequency hits the terahertz range. This regime can be referred as strongly frequency non-degenerate (SFND) PDC.

The SFND regime is interesting with new possibilities that appear in the generation of correlated photons from different spectral ranges, generation of localized long-wave excitations in matter that are bound to optical delocalized states, construction of quantum single-photon sources of terahertz frequencies, quantum calibration of the terahertz wave sources and detectors, and other applications. However, direct transfer of the results and methods, obtained up to now for the PDC in the optical range, to the mixed opticalterahertz case is a quite challenging task. The main problems are caused by the presence of classical thermal field fluctuations at $\mathrm{THz}$ frequencies, inherent absorption of the non-linear medium at $\mathrm{THz}$ frequencies, and high angular diversity of the generated $\mathrm{THz}$ idler photons. Results of the theoretical treatment of correlation functions and frequency-angular distributions of signal and idler photons emitted via spontaneous (low-gain) PDC are presented here accounting the peculiar properties of the SFND regime.

Calculations were made using the nonlinear Kirchhoff law, which expresses the second-order moments of the output PDC-generated fields in terms of the second-order moments of the input fields via elements of the scattering matrix of a nonlinear medi- um. According to its definition, the scattering matrix $\hat{U}$ consists of coefficients that connect linearly firstorder moments of the both signal and idler input and output fields. In particular,

$$
\left\langle a_{s}^{+}(L)\right\rangle=\sum_{s^{\prime}} U_{s s^{\prime}}\left\langle a_{s^{\prime}}^{+}(0)\right\rangle+\sum_{i^{\prime}} U_{s i^{\prime}}\left\langle a_{i^{\prime}}(0)\right\rangle .
$$

Here and below $\left\langle a_{m}(z)\right\rangle$ is considered as an average of the field operator which describes propagation of one plane mode of frequency $\omega_{m}(m=i, s)$ along the layer-cut medium at some angle $\vartheta_{m}$ with respect to the layer's normal. The nonlinear Kirchhoff-type law was formulated by D.N. Klyshko within the logic of fluctuation-dissipation approach for parametric light scattering by polaritons [2]. Applying this approach, one does not need to solve exact Heisenberg equations for field operators with the introduction of special noise operators responsible for the absorbing reservoir. It is enough to consider equations for spatially varying average values of the field operators. These equations have the same structure as the classical wave equations for slowly varying field amplitudes in an absorptive crystal. They were considered accounting the multi-mode character of the parametric interaction caused by a Gaussian transverse-limited pump beam. Explicit expressions for the multimode scattering matrix elements were obtained for the case of spontaneous PDC.

By substituting the scattering matrix elements into the nonlinear Kirchhoff law the second-order moments of the output fields were obtained. The results show that occupations of output modes $\left\langle N_{m}\right\rangle \equiv\left\langle a_{m}^{+}(L) a_{m}(L)\right\rangle$ consist of several different contributions. The first-type contribution (which is true spontaneous) appears due to inherent radiation of the pumped nonlinear medium,

$$
\left\langle N_{s}^{\text {Spon }}\right\rangle=\kappa_{s}\left(\sum_{i}\left|U_{s i}\right|^{2}\right)+\left(\kappa_{s}-1\right)\left(\sum_{i}\left|U_{s i}\right|^{2}\right)\left\langle N_{T}\right\rangle
$$

in each signal mode, and

$$
\begin{aligned}
\left\langle N_{i}^{\text {Spon }}\right\rangle= & \left(\sum_{s}\left|U_{s i}\right|^{2}\right)+\left(1-\kappa_{i}\right)\left(\sum_{s}\left|U_{s i}\right|^{2}\right)\left\langle N_{T}\right\rangle+ \\
& +\left(1-\mathrm{e}^{-2 \mu_{i} L}\right)\left\langle N_{T}\right\rangle
\end{aligned}
$$

in each idler mode. The first terms in Eqs. (2)-(3) contain the pure quantum parts of the nonlinear emission. They are proportional to the sums over all modes of opposite type which make their contributions with the corresponding nonlinear efficiencies $\left|U_{s i}\right|^{2}$, whereas

$$
U_{s i}=i L \gamma_{p s i} \mathrm{e}^{\left(i \Delta k_{\|}-\mu_{i}\right) L / 2} \frac{\sinh \left(i \Delta k_{\|}-\mu_{i}\right) L / 2}{\left(i \Delta k_{\|}-\mu_{i}\right) L / 2} \text {. }
$$


Here, $\mu_{m} \equiv \alpha_{m} / 2 \cos \vartheta_{m}$ depends on the intensity absorption coefficient $\alpha_{m}, \Delta k_{\|}$is a longitudinal projection of the wavevector mismatch, and $\gamma_{p s i} \equiv \frac{2 \pi \omega_{s} \omega_{i}}{c^{2} \sqrt{k_{s z} k_{i z}}} E_{p} \chi^{(2)}$ is a specific gain per unit length which depicts efficiency of the parametric interaction between each three plane modes taken at pump, signal and idler frequencies. $\chi^{(2)}$ is an effective value the medium second-order susceptibility. The pump plane mode components are taken with the amplitudes $\quad E_{p}\left(\mathbf{k}_{p}^{\perp}\right) \sim w_{p}{ }^{2} \exp \left(-w_{p}{ }^{2} \mathbf{k}_{p}^{\perp 2} / 4\right)$ for transverse wave vectors $\mathbf{k}_{p}^{\perp} ; w_{p}$ is a Gaussian beam waist. The angular diversity of the idler modes that are parametrically connected with each optical signal mode was analyzed on the basis of Eq. (4). It was shown, that the solid angle occupied by spontaneously emitted idler photons rapidly increases when the idler wave frequency goes down and the idler wave vector becomes comparable with the allowed wave vector mismatch. However, by proper selection of the transverse $\left(\sim 2 w_{p}\right)$ and longitudinal $(-L)$ dimensions of the interaction volume, the idler radiation divergence can be kept at a moderate level.

The second terms in expressions for spontaneous emission (2)-(3) are responsible for parametric frequency conversion (in Eq.(2)) or amplification (in Eq.(3)) of classical thermal fluctuations at an idler frequency. They are proportional to the mean number of thermal photons $\left\langle N_{T}\right\rangle=\left[\exp \left(\hbar \omega_{i} / k_{B} T_{c r}\right)-1\right]^{-1}$ at a temperature $T_{c r}$ of the nonlinear medium. The lossfactors $\kappa_{s}$ and $\kappa_{i}$ account different influence of $\mathrm{THz}$ absorption on the efficiencies of parametric processes with thermal and quantum field fluctuations. With a high degree of accuracy they can be taken outside the summation procedures as coefficients that depend only on the THz absorption $\mu_{i} L$,

$\kappa_{s}=2\left(\mu_{i} L-1+\mathrm{e}^{-\mu_{i} L}\right) /\left(1-e^{-\mu_{i} L}\right)^{2}, \quad \kappa_{i}=\kappa_{s}\left(-\mu_{i} L\right)$.

In case of negligibly small absorption the nonlinear contributions of thermal fluctuations to the inherent emission of the medium disappear. The last term in Eq.(3) for the number of emitted idler photons describes the non-parametric thermal radiation by the linear Kirchhoff law.

Apart from the spontaneous emission there can be contributions from the signal or idler radiation incident on the medium. For example, if there is any external idler radiation which is characterized by an effective temperature $T_{e x t}$ or with the spectral brightness distributed over all the idler modes with the corresponding mode occupations $\left\langle N_{i}\right\rangle$, the additional photons

$$
\left\langle N_{s}^{\text {Ind }}\right\rangle=\sum_{i}\left|U_{s i}\right|^{2}\left\langle N_{i}\right\rangle
$$

are detected at the output in the signal channel. The method of quantum calibration of spectral brightness of an external terahertz radiation is based on comparison between the spontaneous and induced signals [3]. In case of the pure SPDC registration scheme there are no special sources of the external input radiation. However, the equilibrium radiation of the medium environment should be taken into account.

In most cases the temperatures of the medium and of the environment are the same, and a noticeable contribution of external thermal radiation persists only at idler frequencies. As a result, the detected output signal and idler photon numbers are

$$
\begin{aligned}
& \left\langle N_{s}\right\rangle=\left\langle N_{s}^{\text {Spon }}\right\rangle+\left\langle N_{s}^{\text {Therm.Ind }}\right\rangle=\kappa_{s}\left(\left\langle N_{T}\right\rangle+1\right) \sum_{i}\left|U_{s i}\right|^{2}, \\
& \left\langle N_{i}\right\rangle=\left\langle N_{i}^{\text {Spon }}\right\rangle+\left\langle N_{i}^{\text {Therm.Ind }}\right\rangle=\left(\left\langle N_{T}\right\rangle+1\right) \sum_{s}\left|U_{s i}\right|^{2}+\left\langle N_{T}\right\rangle .
\end{aligned}
$$

Following the nonlinear Kirchhoff law, the second-order moment, which describes correlation of idler and signal fields, is calculated in this case as

$$
\left\langle a_{s}^{+}(L) a_{i}^{+}(L)\right\rangle=\left(\left\langle N_{T}\right\rangle+1\right) \sum_{s^{\prime}} U_{i s^{\prime}} U_{s s^{\prime}} \approx\left(\left\langle N_{T}\right\rangle+1\right) U_{i s^{\prime}} \text {. }
$$

In experimental quantum optics the intensity correlation function

$$
\left\langle I_{s} I_{i}\right\rangle \sim\left\langle: \sum_{s} N_{s} \sum_{i} N_{i}:\right\rangle=\sum_{s, i, s^{\prime}, i^{\prime}}\left\langle a_{s}^{+}(L) a_{i}^{+}(L) a_{s^{\prime}}(L) a_{i^{\prime}}(L)\right\rangle
$$

is frequently measured as an indicator of nonclassicality of the generated field states. Consider two ideal detectors in signal and idler channels connected by a correlation scheme which measures the normalized intensity correlation function. The readings of such scheme should depend on the nonlinear medium parameters as

$$
\frac{\left\langle: \sum_{s} N_{s} \sum_{i} N_{i}:\right\rangle}{\sum_{i}\left\langle N_{i}\right\rangle \sum_{s}\left\langle N_{s}\right\rangle}=1+\frac{\left|\sum_{s, i} U_{i s}\right|^{2}}{\kappa_{s} \sum_{s, i}\left|U_{i s}\right|^{2}}\left(\sum_{s, i}\left|U_{i s}\right|^{2}+\sum_{i}\left\langle N_{T}\right\rangle /\left(\left\langle N_{T}\right\rangle+1\right)\right)^{-1} .
$$

In the case of properly cooled and transparent medium, low gain and low number of involved modes this ratio is sufficiently more than its classical value 1 . However, when absorption, number of modes and the medium temperature are increased, the quantum excess above 1 can become vanishingly small. Final Eq. (9) shows the influence of each of these parameters and can be used as a guide in constructing PDC schemes for generating optical-terahertz biphotons.

The work was done under financial support of the Russian Science Foundation (Grant No. 17-1201134).

\section{References}

1. Bouwmeester D., Ekert A., Zeilinger A. The Physics of Quantum Information: Quantum Cryptography, Quantum Teleportation, and Quantum Computation // New York: Springer. 2000.

2. Klyshko, D.N. Photons and Nonlinear Optics // New York: Gordon and Breach. 1988.

3. Kitaeva, G. Kh., Penin, A. N., Tuchak, A. N., Shepelev, A.V., Yakunin, P. V. Measurement of the spectral brightness of terahertz radiation in the process of nonlinear_optical detection // JETP Lett. 2010. V. 92, No. 5, P. 291-294. 